\title{
Transparent Zinc Oxide Gate Metal-Oxide-Semiconductor Field-Effect Transistor for High-Responsivity Photodetector
}

\author{
Eujune Lee, Dong-Il Moon, Ji-Hwan Yang, Keong Su Lim, and Yang-Kyu Choi
}

\begin{abstract}
We report a new structure of high-responsivity photodetectors that utilizes the transparent and metallic zinc oxide (ZnO) gate in bulk silicon metal-oxide-semiconductor field-effecttransistor photodetectors. The device has a small optical window only in the channel region, and all other regions (depletion) are protected from external light. Whereas the amplification of photocurrent by external light was not significant at the floated or positively biased substrate, the photocurrent was enhanced at the grounded or negatively biased substrate due to the decrement of the recombination rate in the n-channel MOSFET. Responsivity was in excess of $1500 \mathrm{~A} / \mathrm{W}$ under white-light illumination, which is higher than that of conventional photodetectors with the semitransparent polycrystalline-silicon gate.
\end{abstract}

Index Terms-Field-effect phototransistor, photodetector, transparent gate.

\section{INTRODUCTION}

$\mathbf{P}$ HOTODETECTORS are indispensable devices for optoelectronic applications [1]. Compared with compound photodetectors, silicon-based photodetectors have advantages in their monolithic integration with low-cost complementarymetal-oxide-semiconductor (CMOS) technology. CMOS image sensors have attracted much attention due to their advantages of low power consumption, low cost, random sensor access, and design flexibility [2]-[4]. However, low responsivity and nonscalability remain as key disadvantages [5], [6]. Continuous effort has been devoted to enhance sensitivity and integration density [7], [8]. The conventional p-n junction photodiode suffers from a low quantum efficiency [9]. Detection amplification in photodetectors becomes another means to increase sensitivity [10], [11]. A silicon-on-insulator (SOI) metal-oxide-semiconductor field-effect transistor (MOSFET) itself is an example of a highly sensitive photodetector [12], [13], but it still has some constraints [14], [15]. In spite of judicious improvements, such as a gate-body-tied SOI MOSFET with a dynamic threshold MOSFET process [14]

Manuscript received January 29, 2009. First published April 10, 2009; current version published April 28, 2009. This work was supported in part by the National Research and Development Program (NRDP, 2005-01274) for the development of biomedical function monitoring biosensors, sponsored by the Korea Ministry of Education, Science and Technology (MEST). The review of this letter was arranged by Editor C. Jagadish.

The authors are with the School of Electrical Engineering and Computer Science, Division of Electrical Engineering, Korea Advanced Institute of Science and Technology, Daejeon 305-701, Korea (e-mail: ykchoi@ee.kaist.ac.kr).

Color versions of one or more of the figures in this letter are available online at http://ieeexplore.ieee.org.

Digital Object Identifier 10.1109/LED.2009.2016765 or SOI MOSFET-diode composite device [15], there are still inherent shortcomings of using SOI MOSFET photodetectors from the perspective of cost effectiveness.

In this letter, we report the substrate-bias-enhanced photoresponse of a bulk silicon ( $\mathrm{Si}$ ) n-channel MOSFET photodetector with a transparent and metallic $\mathrm{ZnO}$ gate. The field oxide region and the source/drain (S/D) depletion region are completely covered with metal (Al), and only a designed portion of the channel region is exposed to light via the optical window, which is not covered by the metal. Responsivity is enhanced under the substrate bias.

\section{Device Design And Fabrication}

The devices were fabricated on p-type bulk Si (100) wafers. To isolate the device, channel stop doping was performed by boron diffusion at $900{ }^{\circ} \mathrm{C}$ for $20 \mathrm{~min}$, followed by $1-\mu \mathrm{m}$ thick thermal oxide growth to form the field oxide. Channel implantation to make an n-channel was employed with $10-\mathrm{keV}$ boron ions at a dose of $5 \times 10^{12} \mathrm{~cm}^{-2}$, and an S/D junction was formed by implanting $20-\mathrm{keV}$ phosphorus ions at a dose of $2 \times 10^{15} \mathrm{~cm}^{-2}$. A 20 -nm-thick gate oxide was thermally grown. Right after the gate oxidation, a 500-nm-thick zinc oxide layer was deposited by photochemical vapor deposition (photo-CVD) for the transparent and metallic gate. The zinc oxide $(\mathrm{ZnO})$ layer was patterned by the $(1000: 1)$ diluted $\mathrm{HCl}$ wet etchant. A tetraethyl orthosilicate (TEOS) layer of $100 \mathrm{~nm}$ thick was deposited by plasma-enhanced CVD for an interlayer dielectric (ILD) and was then etched to make the contact hole on the gate as well as the S/D. An aluminum layer of $500 \mathrm{~nm}$ thick was deposited by sputtering and patterned to both make an ohmic contact and to cover the S/D depletion region. Another 100-nm-thick layer of TEOS was deposited for the upper ILD. Sequentially, a second aluminum 500-nm-thick layer was deposited to cover the field oxide region. Thereafter, it leaves the optical window only in the channel. As a result, the fabricated devices are entirely covered by double metal layers for blocking external light, except for the optical window that is located on the designed channel region.

Fig. 1 shows a top view of the fabricated device by scanning electron microscopy (SEM) and its schematic cross-sectional view. The device has a small optical window only in the channel region, and all other regions are protected from external light. The optical window is kept away from the depletion region when the drain bias is below $4 \mathrm{~V}$. 

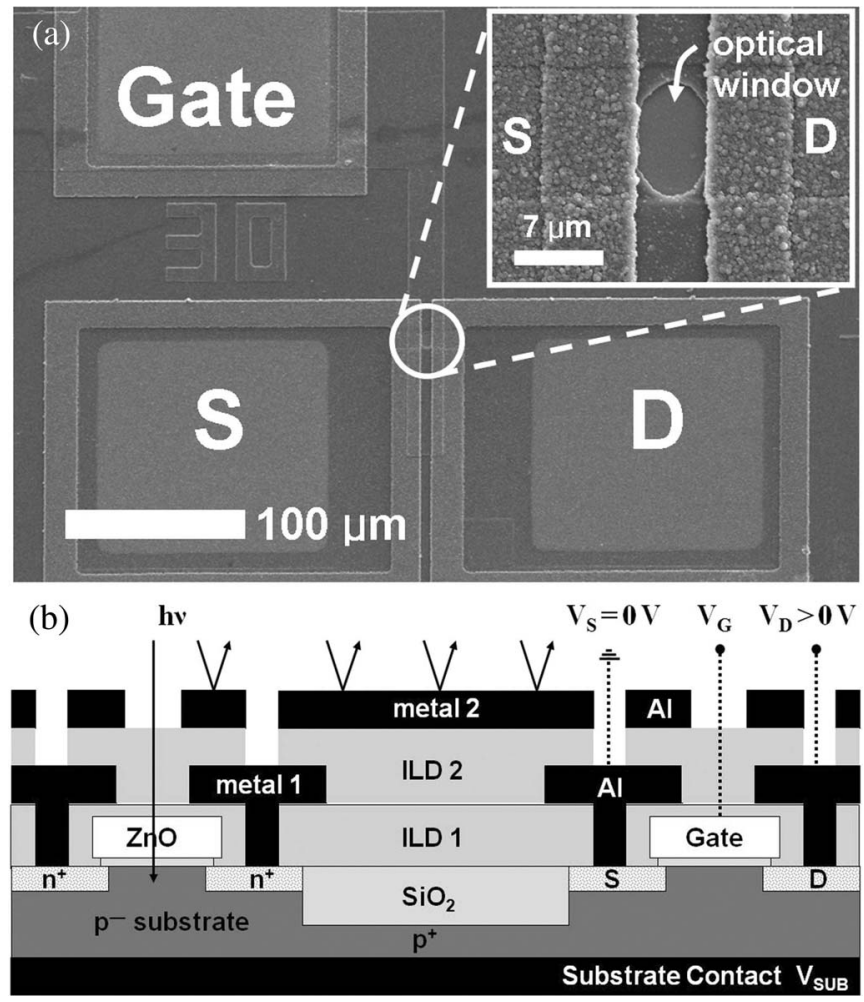

Fig. 1. (a) SEM image of the transparent $\mathrm{ZnO}$ gate $\mathrm{Si}$ photodetector. The optical window exists in the small portion of the channel region. (b) Crosssectional view of the transparent $\mathrm{ZnO}$ gate $\mathrm{Si}$ photodetector.

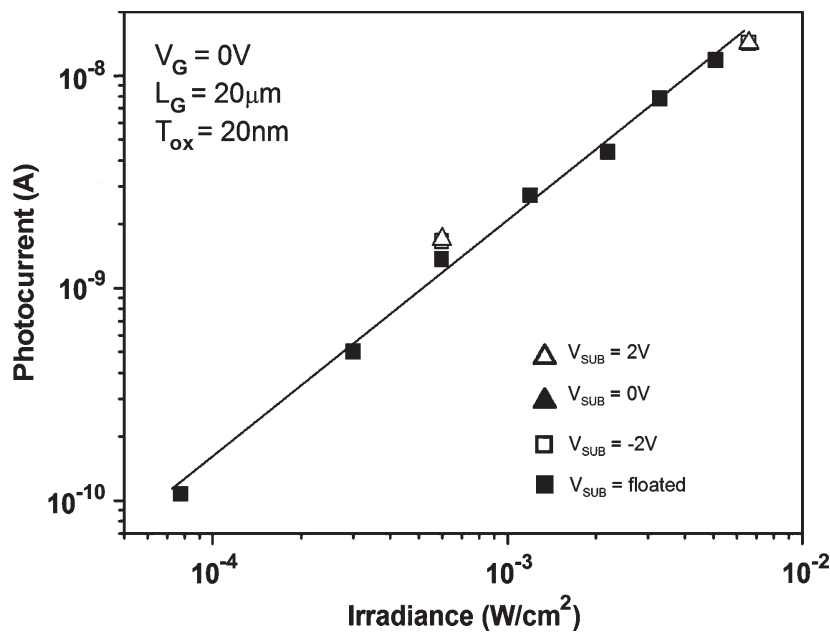

Fig. 2. Photocurrent as a function of irradiance with various substrate biases. $Q_{\mathrm{ph}}$ is irradiance (in watts per square centimeter), and photocurrent (in amperes) is the difference of $I_{D \text {,illum }}$ and $I_{D \text {,dark }}$. Photocurrent is read when the drain bias is $1 \mathrm{~V}$.

\section{RESULTS AND DISCUSSION}

Fig. 2 shows an OFF-state drain photocurrent depending on illumination irradiance. The light source was a halogen lamp. The OFF-state drain photocurrent is linearly proportional to illumination irradiance regardless of the substrate bias. Illuminance corresponding to irradiance was measured, and then, slope $\gamma(=1.46)$ was extracted. The slope is defined as [16]

$$
\gamma=\frac{\partial \log \left(I_{\mathrm{ph}}\right)}{\partial \log \left(Q_{\mathrm{ph}}\right)}=\frac{\partial \log \left(I_{D, \text { illum }}-I_{D, \text { dark }}\right)}{\partial \log \left(Q_{\mathrm{ph}}\right)}
$$
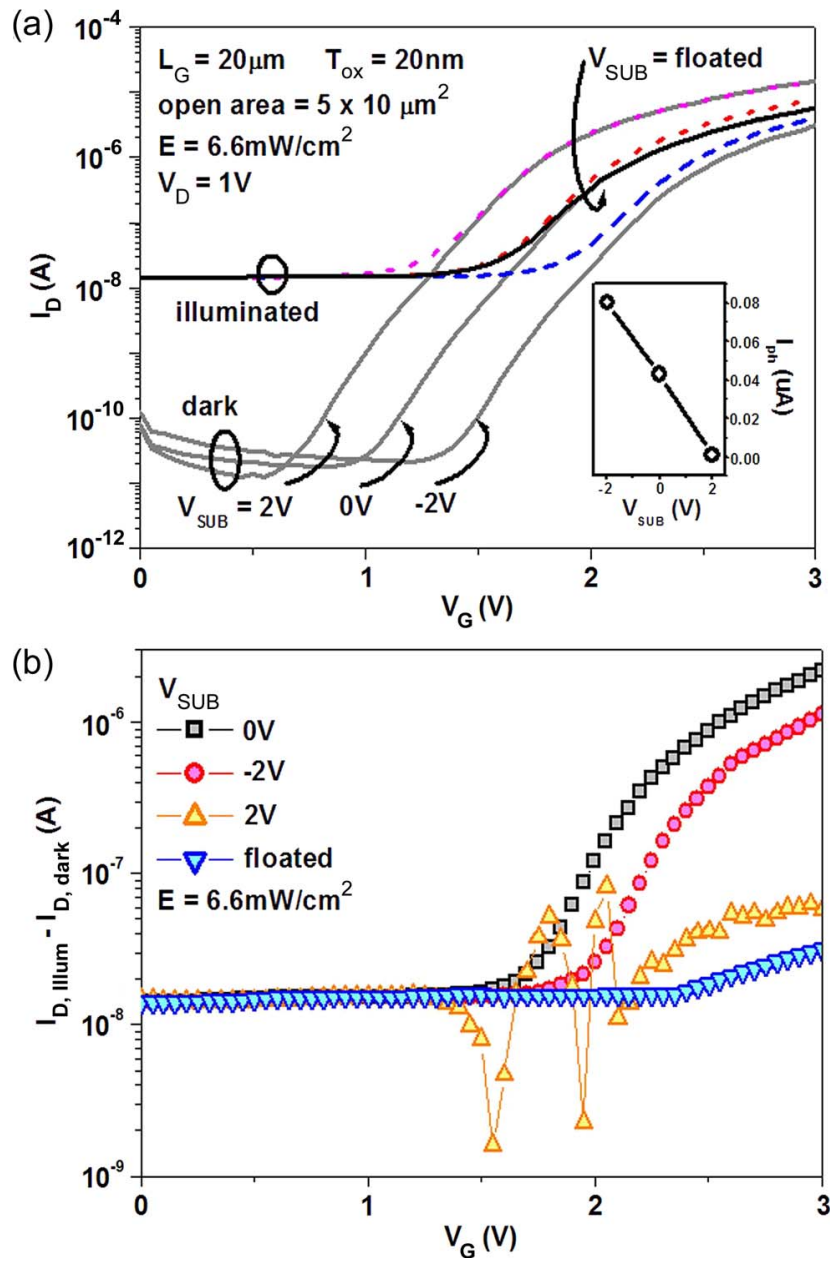

Fig. 3. (a) Transfer characteristics under dark and white-light illumination for the various substrate biases and photocurrent $\left(I_{\mathrm{ph}}\right)$ increment by them. (b) Photocurrent $\left(I_{\mathrm{ph}}\right)$ versus $V_{G}$ for various substrate biases.

where $I_{\mathrm{ph}}$ is the drain photocurrent, $Q_{\mathrm{ph}}$ is illuminance, $I_{D \text {,illum }}$ is the drain current under illumination, and $I_{D \text {,dark }}$ is the drain current without light. This value of $\gamma=1.46$ is satisfactory for application in an image sensor [16].

The transfer characteristics of the device with and without illumination under various levels of the substrate bias are shown in Fig. 3(a). The gate length is $20 \mu \mathrm{m}$. The length and the width of the open area in the channel region are 5 and $10 \mu \mathrm{m}$, respectively. The irradiance is $6.6 \mathrm{~mW} / \mathrm{cm}^{2}$. The OFF-state drain current is strongly dependent on the illumination intensity. When the substrate is floated, $I_{D \text {,illum }}$ in the strong inversion regime does not nearly increase compared with $I_{D \text {,dark }}$. When the substrate is connected to the ground, however, $I_{D \text {,illum }}$ increases in comparison with $I_{D \text {,dark }}$ in the strong inversion regime. The inset of Fig. 3(a) shows $I_{\mathrm{ph}}$ versus $V_{\text {sub }}$. With consideration of the threshold voltage shift by the different substrate biases, the reference gate bias was fixed at $I_{D \text {,dark }}$ of $100 \mathrm{nA}$; thereafter, $I_{D \text {,illum }}$ was read at that gate bias. When the substrate bias is negatively increased, $I_{\mathrm{ph}}$ is accordingly amplified by it, which is similar to the photovoltaic effect [17].

When the gate bias is applied to the $\mathrm{ZnO}$ gate in the floated substrate, there is no significant increase in the drain current by the photocurrent. In contrast, when the gate bias is applied to the $\mathrm{ZnO}$ gate in the biased substrate, clear amplification of 


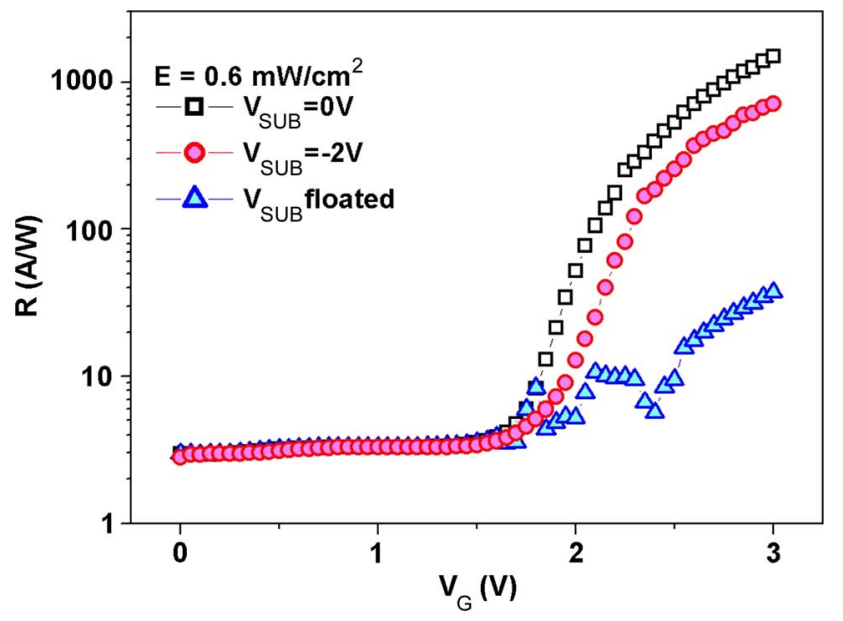

Fig. 4. Responsivity $R$ versus $V_{G}$ for various substrate biases.

the drain current is observed. Moreover, when the substrate is connected to the ground or negative voltage, the generated charges by the light stimulus are separated into holes and electrons; thereafter, holes were swept toward the substrate because of the negative substrate bias. In turn, recombination between the generated electrons and holes can be reduced; hence, the photocurrent was enhanced, as shown in the inset of Fig. 3(a). In the accumulation regime, in which majority of carriers are holes, removing the holes has no effect on the increment of the photocurrent. In the case of the positively biased substrate, no significant amplification of the photocurrent was observed, which is similar to the condition of the floating substrate. This current difference is more clearly shown in Fig. 3(b).

Fig. 4 shows the responsivity-versus-gate-bias curve. Responsivity is defined as [18]

$$
R=\frac{I_{\mathrm{ph}}}{P_{\text {inc }}}=\frac{\left(I_{D, \text { illum }}-I_{D, \text { dark }}\right)}{E \times A}
$$

where $P_{\text {inc }}$ is the power incident on the channel of the device, $E$ is the irradiance of the incident light, $A$ is the effective device area, and the other parameters have been previously defined. Values of $R$ were found to be in excess of $1500 \mathrm{~A} / \mathrm{W}$, which is larger than the previous report [19] in the case of light illumination. This is mainly due to the nature of the transparent zinc oxide $(\mathrm{ZnO})$ gate whose transmittance is over $80 \%$ in the whole visible range, whereas the polycrystalline-silicon gate adopted in conventional MOSFET-type photodetectors absorbs a limited portion of incident photons.

\section{CONCLUSION}

We have fabricated bulk Si MOSFET photodetectors with the transparent and metallic $\mathrm{ZnO}$ gate and demonstrated responsivity in excess of $1500 \mathrm{~A} / \mathrm{W}$ under white-light illumination.
The high responsivity is attributed to improved transmittance of the $\mathrm{ZnO}$ gate compared to the polycrystalline-Si gate. The photocurrent increases in the strong inversion regime under the negative substrate bias. This transparent gate MOSFET can be applicable to optically detect biomolecules.

\section{REFERENCES}

[1] P. Yu, J. Topolancik, and P. Bhattacharay, "Characteristics of a photonic crystal defect waveguide-coupled quantum-dot photodiode," IEEE J. Quantum Electron., vol. 40, no. 10, pp. 1417-1422, Oct. 2004.

[2] M. Schanz, W. Brockherde, R. Hauschild, B. J. Hosticka, and M. Schwarz, "Smart CMOS image sensor arrays," IEEE Trans. Electron Devices, vol. 44, no. 10, pp. 1699-1705, Oct. 1997.

[3] B. Ackland and A. Dickinson, "Camera-on-a-chip," in Proc. ISSCD Tech. Papers, 1996, pp. 22-25.

[4] E. R. Fossum, "Active pixel sensors: Are CCD's dinosaurs?," in Proc. SPIE-Charge-Coupled Devices Solid-State Optical Sensors 111, 1993, vol. 1900, pp. 2-14.

[5] H.-S. Wong, "Technology and device scaling considerations for CMOS imagers," IEEE Trans. Electron Devices, vol. 43, no. 12, pp. 2131-2142, Dec. 1996.

[6] E. R. Fossum, "CMOS image sensors: Electronic camera-on-a-chip," IEEE Trans. Electron Devices, vol. 44, no. 10, pp. 1689-1698, Oct. 1997.

[7] Y. Nagamune, T. Noda, Y. Ohno, Y. Arakawa, H. Sakaki, and M. Watanabe, "Phototransistors using point contact structures," Jpn. J. Appl. Phys., vol. 36, no. 3B, pp. 1955-1957, Mar. 1997.

[8] I. Inoue, N. Tanaka, H. Yamashita, T. Yamaguchi, H. Ishiwata, and H. Ihara, "Low-leakage-current and low-operating-voltage buried photodiode for a CMOS imager," IEEE Trans. Electron Devices, vol. 50, no. 1, pp. 43-47, Jan. 2003.

[9] H. Zimmermann, "Integrated high speed, high sensitivity photodiodes and optoelectronic integrated circuits," Sens. Mater, vol. 13, no. 4, pp. 189 206, 2001.

[10] S. M. Sze, Physics of Semiconductor Devices, 2nd ed. New York: Wiley, 1981, ch. 13, pp. 743-770.

[11] J.-M. Shieh, Y.-F. Lai, W.-X. Ni, H.-C. Kuo, C.-Y. Fang, J. Y. Huang, and C.-L. Pan, "Enhanced photoresponse of a metal-oxide-semiconductor photodetector with silicon nanocrystals embedded in the oxide layer," Appl. Phys. Lett., vol. 90, no. 5, p. 051 105-1, Jan. 2007.

[12] H. Yamamoto, K. Taniguchi, and C. Hamaguchi, "High-sensitivity SOI MOS photodetector with self-amplification," Jpn. J. Appl. Phys., vol. 35, no. 2B, pp. 1382-1386, Feb. 1996.

[13] W. Zhang, M. Chan, S. Fung, and P. K. Ko, "Performance of a CMOS compatible lateral bipolar photodetector on SOI substrate," IEEE Electron Device Lett., vol. 19, no. 11, pp. 435-437, Nov. 1998.

[14] W. Zhang, M. Chan, R. Huang, and P. K. Ko, "High gain gate/body tied NMOSFET photo-detector on SOI substrate for low power applications," Solid State Electron., vol. 44, no. 3, pp. 535-540, Mar. 2000.

[15] Y. Uryu and T. Asano, "SOI-MOSFET/diode composite photodetection device," Jpn. J. Appl. Phys., vol. 40, no. 4B, pp. 2897-2902, Apr. 2001.

[16] Y. Kaneko, N. Koike, K. Tsutsui, and T. Tsukada, "Amorphous silicon phototransistors," Appl. Phys. Lett., vol. 56, no. 7, pp. 650-652, Feb. 1990.

[17] M. A. Romero, M. A. G. Martinez, and P. R. Herczfeld, "An analytical model for the photodetection mechanism in high-electron mobility transistors," IEEE Trans. Microw. Theory Tech., vol. 44, no. 12, pp. 2279 2287, Dec. 1996

[18] M. C. Hamilton, S. Martin, and J. Kanicki, "Thin-film organic polymer phototransistors," IEEE Trans. Electron Devices, vol. 51, no. 6, pp. 877885, Jun. 2004

[19] S.-H. Seo, S.-H. Lee, M.-Y. Do, J.-K. Shin, and P. Choi, "Highly and variably sensitive complementary metal oxide semiconductor active pixel sensor using P-channel metal oxide semiconductor field effect transistortype photodetector with transfer gate," Jpn. J. Appl. Phys., vol. 45, no. 4B, pp. 3470-3474, Apr. 2006 